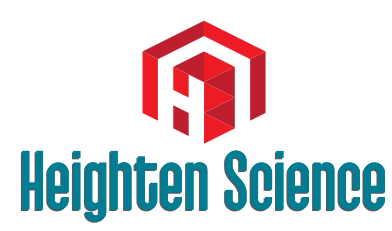

P U B L I C I T I O N S Corporation

\title{
Psychosocial care is necessary in supporting refugees
}

\author{
Gudrun Widders* and Ute Teichert \\ Specialist, General Medicine, Specialist in Public Health in Berlin-Spandau, Berlin, Germany
}

*Address for Correspondence: Gudrun Widders, Specialist in General Medicine, Specialist, Public Health in Berlin-Spandau Gesundheitsamt BerlinSpandau, Carl-Schurz-Straße 4/6, 13597 Berlin, Germany, Tel: +49 17624265265 ;

Email: g.widders@ba-spandau.berlin.de

Submitted: 19 November 2018

Approved: 12 December 2018

Published: 13 December 2018

Copyright: (๑) 2018 Widders G, et al. This is an open access article distributed under the Creative Commons Attribution License, which permits unrestricted use, distribution, and reproduction in any medium, provided the original work is properly cited

Check for updates
Between 2013 and 2016 the main tasks for the German public health care departments were mainly related to medical support in terms of the management of outbreaks of infectious diseases, physical examinations of children and adults to exclude tuberculosis ore other infectious diseases, the administration of vaccinations and medical acute care.

Local health care departments are responsible for the health protection of the entire population. They take care without any difference regarding social status, health insurance affiliation, age and gender. Especially with regards to the evaluation of contact persons in cases of infectious diseases or the organization of appropriate accommodation for these patients the wellbeing of the entire population is in the focus of the public health care system.

There are different specialist areas in German public health care departments: for child and youth health service, social psychiatric service, child and adolescent psychiatric service, service for disabled people and the services for infection protection, hygiene, environmental health protection and medical disaster protection. That's why there are different tasks with regards to refugees.

While the number of refugees rose from 5.700 to 45.000 from 2013 to 2016, in 2018 about 22.300 refugees will live in Berlin in shared accommodation. Although the number of refugees is declining, the engagement of local health care departments is still the same. However, the psychosocial care is currently the main focus.

Refugees are now receiving regular medical care. Large emergency shelters were dissolved. Refugees now live in shared accommodation or apartments. Infection outbreaks have become rare. But traumas suffered in the home country or on the run have remained with most people. Also, accommodation in an emergency shelter over a long period of time can cause traumatization. Another negative factor is a lack of professional prospects or an unsuccessful integration into society. Therefore psychosocial care is needed.

What are practical experiences of the health care department in BerlinSpandau?

Current reports in the media include exactly those things which are expected, if no preventive action is taken: addictions, mental illnesses, violence and suicidality. They even report about injured, raped and killed people. The social-psychiatric services frequently deal with traumatized refugees, people with mental illness or addictions and people, which are aggressive or suicidal. Also the number of violent refugees in psychiatric departments has been reported to rise. Authorities have to take care of an increasing number of funerals of refugees who committed a suicide. This was seen while the work in the social psychiatric service, in the refugee facilities, in the 
psychiatric clinic of the district and in the regulatory authorities for burial burials of persons without relatives.

Especially people with traumatic experiences have an increased risk to develop mental illness. Also many refugees were accommodated in emergency shelters for a long time.

Many of them cannot expect an own home, an education or job opportunity. Also many refugees still wait for relatives and friends from their home countries. All of these people who are not primarily mentally sick are not recognized by the regular health care system. But they either have an increased risk to develop mental illness or have already mental illness, which was previously undetected. Although accommodation conditions have been improved for most refugees traumatization are still present and are likely to have an impact on life planning and long term perspective.

\section{What are the consequences?}

If mental illness or addictions are not targeted preventively and threatening situations cannot be detected in time substantially increasing costs are expected and a damage of previously uninvolved people cannot be ruled out. If integration fails, this will put significant cost to society because the chance was not taken to educate qualified employees and to create active members of the society. Instead, costs for social welfare and medical support will be raised.

Possible consequences for all affected people themselves are traumatization again, development of mental illness and transfer of vulnerability to subsequent generations. Children of healthy people grow up differently compared to children of healthy people. Illness or even suicide could be prevented, when proactive action can be taken.

\section{What is needed?}

Local contacts are important to gain trust and confidence, to support integration in the regular health care system. Psychiatric intervention is unnecessary in most cases. But often refugees are afraid of disintegration and cannot accept on-site psychiatric offers.

Foreigners who are not familiar with the structure of our society and with our language need permanent support to develop self-confidence by people who are appropriately educated to detect risks for mental illnesses and which are therefore able to take competently preventive action. It is necessary that these are constantly on site. There is a need for low-threshold social-educational and psychological services on site as well as for psychiatric, psychotherapeutic and psychological care. These findings are the result of the experiences of a large shelter with over 1.000 beds in which these offers were submitted. There are also experiences of the psychiatric institute outpatient department of the district and of the social-psychiatric service of the health department.

\section{What is specifically needed?}

\section{Urgently needed:}

- Continuous low-threshold presence (trusted persons with professional qualifications)

- Psychological or psychotherapeutic offers on site in institutions and dwellings, an escort service in this regard

- Qualified support in terms of the handling of day by day issues

- Qualified social work with appropriate head count 
- Training, multiplier training

- Peer Groups

- Professional advice and support

Generally, medical support must be provided for all members of our society for ethical reasons. As long as this support cannot be provided by the regular health care system, the Public health care system will be in charge. Special attention must be paid on prevention of psychiatric diseases, addictions, violence or suicidality. This preventive action is important to improve the long term perspective of refugees in our country but also to maintain the quality of life of the local population. A legal framework must be established and an appropriate financial basis must be in place.

It is the responsibility of the entire society to integrate refugees and to provide a long term perspective of a self-determined life. This includes education, appropriate accommodation, participation in our culture and interaction into the social environment 